Philippe Roingeard - Histopathology 2013 ; 61, 991-993.

\title{
Quantifying hepatic steatosis by electron microscopy
}

Sir : Given the increasing number of patients with non-alcoholic fatty liver disease (NAFLD), Levene and collaborators have pointed out in a recent issue of Histopathology the importance of quantifying the steatosis accurately in a liver biopsy. ${ }^{1}$ In this study, the Oil Red-O (ORO) staining of frozen liver sections studied by light microscopy and digital image analysis (DIA) was the most accurate method for detecting and quantifying steatosis. The authors demonstrated that the ORO DIA method was more accurate that the current visual assessment of haematoxylin and eosin (H\&E) staining by pathologists to determine the percentage of hepatocytes with fat, or an approach combining H\&E staining and DIA. However, if the ORO DIA method was able to appreciate the fat deposition in liver sections with either macrovesicular or microvesicular steatosis, the images provided in this study showed that the lipid droplets (LD) were highly deformed and had probably fused during the sample preparation. Thus, the ORO DIA method may be not the optimal method to monitor the number and size of LD in liver sections.

In previous investigations on cultured cells producing various wild-type or mutated hepatitis $\mathrm{C}$ virus (HCV) core proteins, known to induce LD accumulation, my group has established an original method for quantifying the steatosis by transmission electron microscopy (TEM). ${ }^{2}$ More recently, we applied this method to the analysis of liver biopsy specimens from HCV chronic carriers, allowing to quantify precisely the accumulation of the LD in liver tissues, even when the LD are small (Figure 1). ${ }^{3}$ In this method, LD diameter is determined on the computer screen for five consecutive squares $\left(5000 \mu \mathrm{m}^{2}\right.$ each) of two independent TEM grids (i.e. 10 different areas of $5000 \mu \mathrm{m}^{2}$ of hepatic tissue, for each biopsy). LD diameter is then converted into a disk area in $\mu \mathrm{m}^{2}$. The areas covered by the LD over which a TEM grid bar is partly superimposed is determined specifically with the Image $\mathbf{J}$ program (National Institute of Health, USA). By summing these areas, it is then possible to determine the cumulative LD area in $\mu \mathrm{m}^{2}$ per TEM grid square. For each biopsy, the areas in the 10 squares analyzed are then summed, allowing the precise quantification of steatosis in the liver tissues of the patients. The analysis of the number and the mean diameter of the LD encountered in $50,000 \mu^{2}$ of liver tissue can be also performed with this approach. ${ }^{3}$

As for the ORO staining, the analysis and quantification of the hepatitic steatosis by TEM is certainly not practical for a routine use on a large number of patients. However, a precise determination of LD number and size in liver samples by TEM could be of great 
Philippe Roingeard - Histopathology 2013 ; 61, 991-993.

interest in future research protocols in human or experimental animal models in which changes in the steatosis status should be precisely monitored.

\section{Philippe Roingeard}

INSERM U966, Université François Rabelais and CHRU de Tours, Tours, France (roingeard@med.univ-tours.fr)

1. Levene AP, Kudo H, Armstrong MJ, et al. Quantifying hepatic steatosis - more than meets the eye. Histopathology 2012 ; Feb 28. doi: 10.1111/j.1365-2559.2012.04193.x. [Epub ahead of print]

2. Hourioux C, Patient R, Morin A, et al. Hepatitis C virus core protein residue phenylalanine 164 increases steatosis in an in vitro cellular model. Gut 2007 ; 56 ; 1302-1308.

3. Depla M, d'Alteroche L, Le Gouge A, et al. Viral sequence variation in chronic carriers of hepatitis C virus has a low impact on liver steatosis. PLoS One 2012 ; 7 ; e33749.
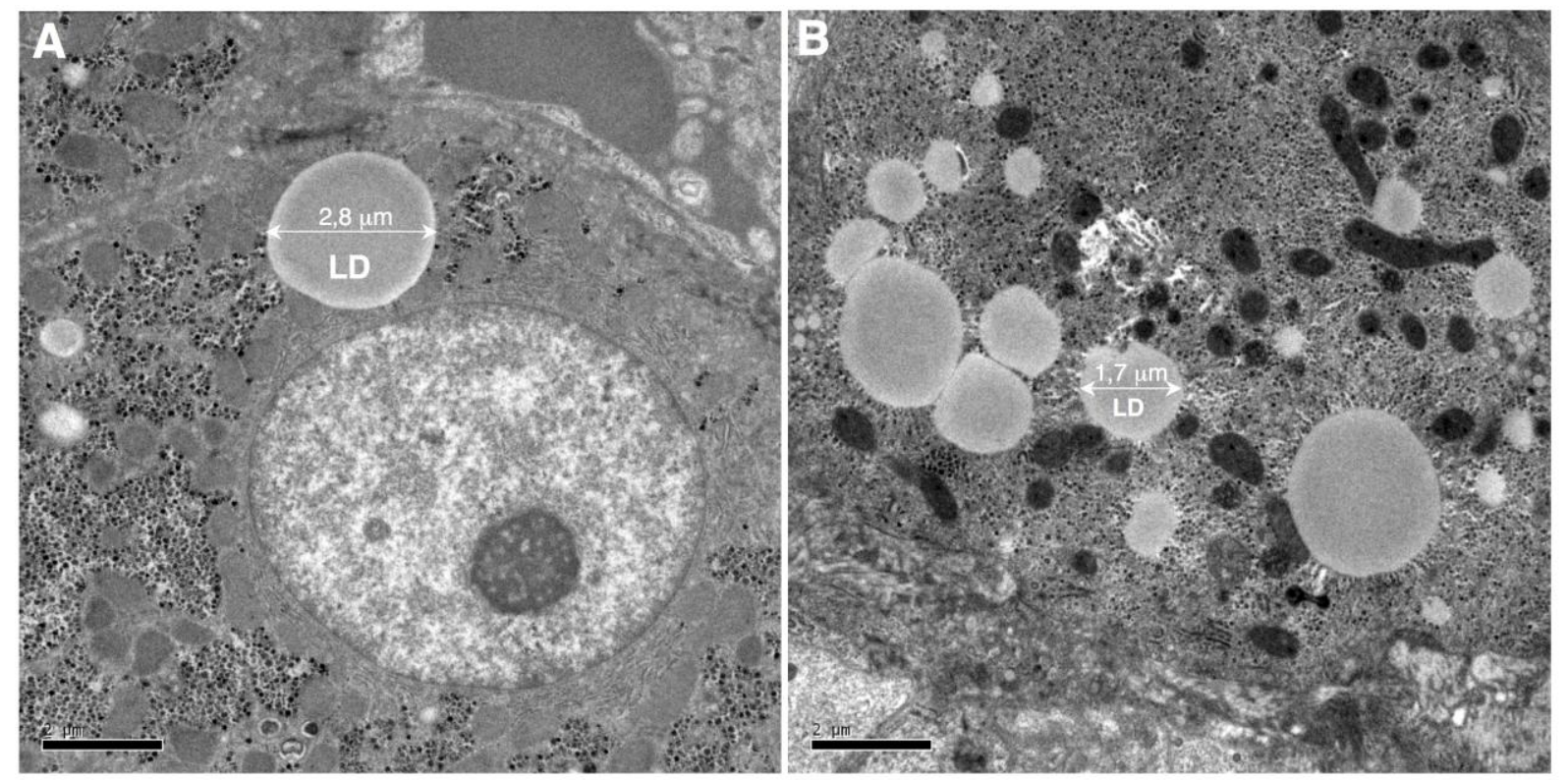

Figure 1. TEM observation of two liver biopsy specimens (A \& B) from two different patients chronically infected by HCV. The diameter of the lipid droplets (LD) is determined on the computer screen and this diameter is then converted into a disk area in $\mu \mathrm{m}^{2}$. By summing the areas of all LD in several TEM grid squares, it is possible to determine the cumulative LD area in $\mu \mathrm{m}^{2}$ in a given surface of liver tissue. 